\title{
1000 sample comparison of MLPA and RT-PCR for carrier detection and diagnostic testing for Spinal Muscular Atrophy Type 1
}

\author{
Charles M. Strom ${ }^{1}$, Ben Anderson ${ }^{1}$, Mei Peng $^{1}$, Urjit Patel ${ }^{2}$, Corey D. Braastad ${ }^{2}$, Weimin Sun ${ }^{1}$ \\ ${ }^{1}$ Quest Diagnostics Nichols Institute, San Juan Capistrano, USA \\ ${ }^{2}$ Athena Diagnostics, Worcester, USA \\ Email: ${ }^{\text {Charles.M.Strom@questdiagnostics.com }}$ \\ Received 24 January 2013; revised 28 February 2013; accepted 1 May 2013 \\ Copyright (C) 2013 Charles M. Strom et al. This is an open access article distributed under the Creative Commons Attribution License, \\ which permits unrestricted use, distribution, and reproduction in any medium, provided the original work is properly cited.
}

\begin{abstract}
Purpose: To compare the accuracy of a commercially available MLPA kit with a laboratory developed RT-PCR assay for the detection of SMN1 and SMN2 copy numbers in clinical samples. Methods: We developed and validated a laboratory developed real time PCR based test capable of detecting SMN1 and SMN2 copy numbers in individuals. We also validated an MLPA kit purchased from MRC Holland for the same purpose. We then analyzed a series of 1027 anonymized samples using both technologies. When discrepant results were obtained, each sample was re-analyzed at least twice using both platforms. Results: Five samples did not yield results in either assay. For SMN1 copy number analysis, 2 RT-PCR assays revealed indeterminant results and all 1020 other samples were concordant for SMN1 copy number. There were 9 discrepancies in SMN2 copy number determination mostly due to a variability in MLPA analysis. Conclusion: Both MLPA and RTPCR assays give a reliable estimate of SMN1 copy number and are therefore appropriate for population based carrier screening for Spinal Muscular Atrophy Type 1. The MLPA kit has a low incidence $(<1 \%)$ of underestimating the SMN2 copy number by 1 copy, but this inconsistency is of little clinical significance and can be overcome by replicate testing.
\end{abstract}

Keywords: Spinal Muscular Atrophy; Carrier Screening; SMN1; SMN2; MLPA

\section{INTRODUCTION}

Spinal Muscular Atrophy Type 1 (SMA-1, OMIM \#

\footnotetext{
"Corresponding author.
}

253300) or Werdnig-Hoffman disease is an autosomal recessive neurodegenerative disorder with an estimated incidence of 1:10,000 live births. The disease causes degeneration of motor neurons in the anterior horn of the spinal canal resulting in progressive atrophy of skeletal muscles thus leading to disability and eventually death from respiratory insufficiency. There are 4 recognized forms of SMA (I-IV) with the most severe being SMA1 having the earliest onset and the most rapid progression. The carrier frequency of SMA-1 is estimated to be 1:40 to $1: 60$ in the US [1-6].

Ninety-five percent of SMA1 cases are due to homozygous deletions of exon 7 of the survivor motor neuron 1 (SMN1) gene. The remainder of patients is compound heterozygotes for a deletion on one chromosome and deleterious point mutation or small insertion or deletion on the other. A closely related gene, SMN2, exists proximal to the SMN1 gene on chromosome 5q. There are only 5 base pair differences between SMN1 and SMN2 and are all synonomous mutations. The exon 7 base changes in SMN2 causes alternative splicing with skipping of exon 7 in the mature transcript leading to reduced levels of active protein. There are individual variations in the number of SMN2 genes. In general, in affected patients, a greater number of SMN2 genes lessen the severity of the phenotype. In 2008, the American College of Medical Genetics (ACMG) recommended that population based carrier screening for SMA be offered to all couples of reproductive age [7].

The identification of carriers of SMA relies on determining the copy number of exon 7 of the SMN1 gene. Individuals with 2 or more copies of exon 7 of SMN1 are considered to be non-carriers of SMA. This approach will not detect all carriers of SMA. Some carrier individuals will harbor point mutations or small insertions/deletions in an SMN1 gene and some individuals 
will have 2 syntenic copies of SMN1 and a deleted copy on the other chromosome (the $2+0$ genotype). Individuals with a single copy of exon 7 of SMN1 gene are carriers of SMA. Ninety five percent of affected individuals will have zero copies of SMN1.

There are 2 established methods for determination of SMN1 exon 7 copy number [8], Multiplex Ligation Dependant Probe Amplification (MLPA) [9] and quantitative fluorescent real time PCR [10]. In this study, we compared the performance of these 2 assays in detecting copy numbers of exon 7 of both SMN1 and SMN2 genes. Prior to introducing a new platform into our laboratory we routinely perform a 1000 sample comparison in order to determine the accuracy of the new platform [11-13]. In this case we performed a 1000 sample comparison of both methods. This study determined that both methods are capable of identifying the SMN1 exon 7 copy number and are therefore acceptable for the purposes of carrier screening, but the determination of SMN2 copy number was somewhat more problematic.

\section{MATERIALS AND METHODS}

\subsection{Subjects}

Anonymized residual DNA samples were used from whole blood specimens submitted for SMA testing at Nichols Institute (San Juan Capistrano, CA) between the months of February and April in 2012. Samples were initially analyzed using the MLPA kits purchased from MRC Holland. Samples were de-identified with the exception of the SMN1 and SMN2 exon 7 copy numbers. This study was determined to be exempt from requiring consent by the Western Institutional Review Board.

\subsection{DNA Isolation}

Genomic DNA was isolated using the MagNA Pure 96 DNA and Viral NA small volume kit (Roche, Indianapolis, IN) on the MagNA Pure 96 extraction instrument (Roche, Indianapolis, IN) as described by the manufac- turer. DNA was not quantified before use.

\subsection{Allele-Specific Real Time PCR}

Allele specific real-time PCR was performed with modification as described previously (1). A fragment from the Cystic Fibrosis Conductance Regulator (CFTR) gene was co-amplified as an endogenous control. Both the SMN and CFTR probes were MGB TaqMan probes (Life Technologies, Foster City, CA). The PCR reactions for SMN1 and SMN2 are carried out in two separate reactions. Table 1 is a listing of the primers used in this assay. The PCR conditions for both the SMN1 and SMN2 reactions are as follows: 1X TaqMan Fast Advanced Master Mix (Life Technologies, Foster City, CA), $900 \mathrm{nM}$ SMN1 or SMN2 forward and SMN reverse primers, 800 $\mathrm{nM}$ CFTR primers, $250 \mathrm{nM}$ of both SMN and CFTR probes. The PCR reaction for SMN2 also includes a nonextending oligonucleotide (600 $\mathrm{nM}$ final concentration) that is used to block the amplification of SMN1. It was determined during assay development that a non extending oligonucleotide for SMN2 was not needed in the SMN1 PCR reaction.

The real-time PCR was performed using the ViiA7 System (Life Technologies, Carlsbad, CA). Samples were run in quadruplicate in a 384 well plate using the comparative $\mathrm{C}_{\mathrm{T}}\left(\Delta \Delta \mathrm{C}_{\mathrm{T}}\right)$ method. Standard ViiA7 thermal cycling conditions were used which was, $50^{\circ} \mathrm{C}$ for $2: 00$, $95^{\circ} \mathrm{C}$ for $10: 00$, then 40 cycles of $95^{\circ} \mathrm{C}$ for $0: 15$ and $60^{\circ} \mathrm{C}$ for 1:00.

Data was analyzed using the ViiA7 Software using standard manufacturer's analysis settings. A control with 2 copies of SMN1 and SMN2 was chosen as the reference sample. Allele determinations were as follows: ratios between $0-0.2,0$ copy; between $0.4-0.6,1$ copy; between 0.8 - 1.2, 2 copies, between 1.3 - 1.65, 3 copies; greater than $1.7,4+$ copies. Any sample with a value between those limits was repeated. The range for 1 copy for SMN2 was $0.4-0.7$, with all other ranges being the same as that of SMN1.

Table 1. PCR primers and probe sequences for real time PCR assay.

\begin{tabular}{cc}
\hline SMN1-F & TTCCTTTATTTTCCTTACAGGGTGTC \\
SMN2-F & TTCCTTTATTTTCCTTACAGGGTGTT \\
SMN-R & GCTGGCAGACTTACTCCTTAATTTAA \\
CF17-F & AGCCGACACTTTGCTTGCTATG \\
CF17-R & GACATAGGTGCTTGAAGAACAGAATG \\
SMN-Probe & 6FAM-ACCAAATCAAAAAGAAGGAAGGTGCTCACA-MGBNFQ \\
CF17-Probe & VIC-TCAGAGGTCTACCACTGGTGCATACTC-MGBNFQ \\
SMN1-blocker & ATTTTCCTTACAGGGTTTCAGACAAAATCAAAA-3Phos \\
\hline
\end{tabular}




\subsection{MLPA Analysis}

The MLPA assay was performed using the SALSA MLPA kit P060-B1 (MRC Holland, Amsterdam, the Netherlands) following the manufacturers directions. The MLPA products were detected using the Applied Biosystems 3100 Genetic Analyzer (Life Technologies, Foster City, CA) and analyzed using a combination of GeneMapper Analysis Software (Life Technologies, Foster City, CA) and SeqPilot Software (JSI Medical Systems, Germany). The SeqPilot MLPA analysis module, P060B2, was downloaded from JSI Medical Systems.

\section{RESULTS}

All but 5 samples were successfully genotyped by both platforms. The 5 samples failed analysis by both platforms. The results for the 1022 samples with results for both platforms, 1016 results were concordant for both SMN1 and SMN2 copy number (99\%). When a discrepancy was noted the samples were repeated using both methods. If that did not resolve the issue, further work was performed. Table 2 is a summary of the genotyping results for this series of patients.

There were 2 discrepant results for SMN1 gene quantitation. On the RT-PCR assay, one sample repeatedly appeared to have an SMN1 copy number between 1 and 2. The MLPA analysis for this individual was consistently 2 copies. DNA sequencing revealed this patient had a rare polymorphism in exon 7 within the primer sequence for the RT-PCR assay. A second individual consistently demonstrated a copy number between 2 and 3 on the RT-PCR assay and 3 copies by the MLPA assay. No further analysis was done on this sample.

Nine samples were discrepant for SMN2 copy number. In 6 of the 9 samples, RT-PCR revealed 2 copies of SMN2 whereas the initial MLPA result was 3 copies. Repeat MLPA analysis revealed 2 copies of SMN2. One sample had an RT-PCR result of 3 copies and an initial MLPA result of 2 copies. The second MLPA analysis detected 3 copies of SMN2. In 2 samples the discrepancies could not be resolved by repeat analysis. In both individuals, the RT-PCR results was indeterminant, between 1 and 2 copies in the first case and between 2 and

Table 2. Genotyping results for 1022 consecutive individuals.

\begin{tabular}{ccc}
\hline Copy Number & SMN1 & SMN2 \\
\hline 0 & 1 & 81 \\
1 & 18 & 443 \\
2 & 885 & 481 \\
3 & 103 & 17 \\
$\geq 4$ & 15 & 0 \\
\hline
\end{tabular}

3 copies in the second case. MLPA consistently revealed 2 copies of the SMN1 gene. Since the SMN2 copy number has no relevance to carrier detection, no further investigation of these cases was undertaken.

There were 18 carriers for SMA in this series of 1022 patients for a prevalence of 1:56. This is within the expected range of 1:40 - 1:60. We did not collect ethnicity for any of these samples. Of note, was that 103 individuals (approximately 10\%) had 3 SMN1 genes indicating that one of their chromosomes likely had a duplication of the SMN1 gene $(2+1$ genotype). SMA carriers who have a duplicated chromosome and a deleted chromosome $(2+0)$ will have false negative results on carrier screening using both of these modalities. This is also within expected levels for a pan-ethnic population.

As expected, SMN2 copy number variants are more prevalent than SMN1 variants in this study. In fact only $47 \%$ of all individuals had 2 copies on the SMN2 gene (481 of 1022, see Table 2). An almost equal number $(43 \%)$ of individuals had only 1 SMN2 gene.

\section{DISCUSSION}

Samples from 1016 consecutive patients were analyzed by MLPA and real time PCR. There were 5 samples that failed analysis by both platforms $(0.5 \%)$ indicating that there was likely insufficient DNA isolated from these samples. Both platforms were able to accurately determine the copy number of exon 7 of the SMN1 gene. In 2 individuals the RT PCR assay revealed levels outside of accepted binning values. In one sample the value was between 1 copy and 2 copies. This was due to a rare polymorphism occurring under one of the PCR primers. If this sample was encountered in clinical testing it would have been reported out as indeterminant. MLPA testing revealed that this sample actually had 2 copies of exon 7 of SMN1. The second sample had a value between 2 and 3 copies on the RT PCR assay. This could have been reported out as having at least 2 copies of exon 7 of SMN1 and therefore not a carrier. From this experience, we can intuit that when a real time PCR result falls between our binning parameters, the cause is likely from a polymorphism and MLPA analysis can be performed to confirm this interpretation.

The determination of SMN2 copy is more problematic. Although $99 \%$ of samples were accurately determined by both platforms, there were problems with reproducablility of the MLPA assay in 7 cases. In 6 of the seven cases, the initial MLPA analyses overestimated the copy number as 3 when it was actually 2 . In a seventh case the initial MLPA analysis revealed 2 copies of SMN2 whereas repeated assays consistently confirmed the RTPCR result of 2 copies. In 2 cases the RT-PCR results were indeterminant, whereas the MLPA results were 
diagnostic.

These data demonstrate that both the RT-PCR and MLPA assays are acceptable for determining the exon 7 copy number of the SMN1 gene and therefore would be suitable for population based carrier screening. In the rare occurrence $(0.2 \%)$ of an indeterminant RT-PCR result, MLPA can used to determine SMN1 copy number. Because of the $10 \%$ prevalence of chromosomes with 2 copies of SMN1, this testing would be expected to have a false negative rate of approximately $10 \%$. Other modalities of testing will need to be developed in order to detect to $2+0$ genotype in carrier screening.

Accurate determination of SMN2 copy not number is not required for population based carrier screening, but has relevance to prognosis in diagnostic testing for affected individuals. Our data would suggest that in rare cases $(0.6 \%)$ the MLPA assay has some variability in results and should probably be performed at least 2 times in order to assure an accurate result.

\section{REFERENCES}

[1] Lefebvre, S., Burglen, L., Reboullet, S., et al. (1995) Identification and characterization of a spinal muscular atrophy-determining gene. Cell, 80, 155-165. doi:10.1016/0092-8674(95)90460-3

[2] McAndrew, P.E., Parsons, D.W., Simard, L.R., et al. (1997) Identification of proximal spinal muscular atrophy carriers and patients by analysis of SMNT and SMNC gene copy number. The American Journal of Human Genetics, 60, 1411-1422. doi:10.1086/515465

[3] Mailman, M.D., Heinz, J.W., Papp, A.C., et al. (2002) Molecular analysis of spinal muscular atrophy and modification of the phenotype by SMN2. Genetics in Medicine, 4, 20-26. doi:10.1097/00125817-200201000-00004

[4] Ogino, S. and Wilson, R.B. (2002) Genetic testing and risk assessment for spinal muscular atrophy (SMA). $\mathrm{Hu}$ man Genetics, 111, 477-500.

doi:10.1007/s00439-002-0828-x
[5] Pearn, J. (1978) Incidence, prevalence, and gene frequency studies of chronic childhood spinal muscular atrophy. Journal of Medical Geneticst, 15, 409-413. doi:10.1136/jmg.15.6.409

[6] Wirth, B., Rudnik-Schoneborn, S., Hahnen, E., Rohrig, D. and Zerres, K. (1995) Prenatal prediction in families with autosomal recessive proximal spinal muscular atrophy (5q11.2-q13.3): Molecular genetics and clinical experience in 109 cases. Prenatal Diagnosis, 15, 407-417. doi:10.1002/pd.1970150503

[7] Prior, T.W. (2008) Carrier screening for Spinal Muscular Atrophy. Genetics in Medicine, 10, 840-842. doi:10.1097/GIM.0b013e318188d069

[8] Prior, T.W., Nagan, N., Sugarman, E.A., Batish, S.D. and Braastad, C. (2011) Technical standards and guidelines for spinal muscular atrophy testing. Genetics in Medicine, 13, 686-694. doi:10.1097/GIM.0b013e318220d523

[9] (1 September 2012) http://www.mlpa.com/

[10] Gomes-Curet, I., Robinson, K.G., Funanage, V.L., Crawford, T.O., Scavina, M. and Wang, W. (2007) Robust quantification of the SMN gene copy number by realtime Taqman PCR. Neurogenetics, 8, 271-278. doi:10.1007/s10048-007-0093-1

[11] French, C., Li, C., Strom, C. and Sun, W. (2004) Detection of factor $\mathrm{V}$ Leiden mutation by a modified photo cross-linking oligonucleotide hybridization assay. Clinical Chemistry, 50, 296-305. doi:10.1373/clinchem.2003.023556

[12] Strom, C.M., Janeczko, R.A., Anderson, B., Redman, J., Quan, F., Buller, A., McGinniss, M. and Sun, W. (2005) Technical validation of a multiplex platform to detect 30 mutations in 8 genetic diseases prevalent in individuals of Ashkenazi Jewish descent. Genetics in Medicine, 7, 633639. doi:10.1097/01.gim.0000187120.93597.16

[13] Strom, C.M., Janeszco, R., Quan, F., Wang, S.-B., Buller, A., McGinniss, M. and Sun, W. (2006) Technical validation of a Luminex based multiplex assay for 40 mutations in the cystic fibrosis transmembrane regulator protein. Journal of Molecular Diagnostics, 8, 371-375. doi:10.2353/jmoldx.2006.050115 